\title{
Anti-inflammatory management for tendon injuries - friends or foes?
}

\author{
Kai-Ming Chan and Sai-Chuen Fu*
}

Address: Department of Orthopaedics and Traumatology, Faculty of Medicine, The Chinese University of Hong Kong, Hong Kong, PR China

Email: Kai-Ming Chan - kaimingchan@cuhk.edu.hk; Sai-Chuen Fu* - bruma@cuhk.edu.hk

* Corresponding author

Published: 13 October 2009

Sports Medicine, Arthroscopy, Rehabilitation, Therapy \& Technology 2009, I:23 doi: 10.1 I86/I 758-2555-I-23

This article is available from: http://www.smarttjournal.com/content/I/I/23

(C) 2009 Chan and Fu; licensee BioMed Central Ltd.

This is an Open Access article distributed under the terms of the Creative Commons Attribution License (http://creativecommons.org/licenses/by/2.0), which permits unrestricted use, distribution, and reproduction in any medium, provided the original work is properly cited.
Received: 10 September 2009

Accepted: 13 October 2009

\begin{abstract}
Acute and chronic tendon injuries are very common among athletes and in sedentary population. Most physicians prescribe anti-inflammatory managements to relieve the worst symptoms of swelling and pain, including non-steroidal anti-inflammatory drugs, corticosteroids and physical therapies. However, experimental research shows that pro-inflammatory mediators such as prostaglandins may play important regulatory roles in tendon healing. Noticeably nearly all cases of chronic tendon injuries we treat as specialists have received non-steroidal anti-inflammatory drugs by their physician, suggesting that there might be a potential interaction in some of these cases turning a mild inflammatory tendon injury into chronic tendinopathy in predisposed individuals. We are aware of the fact that non-steroidal anti-inflammatory drugs and corticosteroids may well have a positive effect on the pain control in the clinical situation whilst negatively affect the structural healing. It follows that a comprehensive evaluation of anti-inflammatory management for tendon injuries is needed and any such data would have profound clinical and health economic importance.
\end{abstract}

Sporting injuries, including both acute trauma and chronic overuse, are usually presented with clinical signs of swelling and pain. Most sport medicine physicians prescribe anti-inflammatory managements to control the pain. However, there are increasing evidences about the unfavorable side effects on the use of various anti-inflammatory agents for tendon injuries.

\section{Do we need to re-visit the rationale of this practice?}

In cases of chronic tendinopathy, a lack of inflammatory infiltration in the biopsies has challenged the status of "tendinitis". However, it did not rule out the possibility of an inflammatory response in the early development of tendinopathy. Overuse or repetitive stretching on tendons triggered the release of pro-inflammatory mediators, which can induce expression of metalloproteinases and leads to collagen degradation. These findings suggest that inflammatory responses play a key role in the development of degenerative overuse tendon injuries. Together with the fact that all chronic tendinopathy cases received anti-inflammatory management, it is logical to assume that suppression of inflammatory responses may interact with the failed healing of degenerative tendon injuries. We coined the term failed healing to describe the histopathological characteristics of tendinopathy samples, which exhibited traits of both active repair and degenerative injuries. The development of overuse animal model clearly demonstrated the casual relationship of overuse and degenerative tendon injuries through activation of inflammatory mediators [1], but whether the injured tendons failed to heal in these models were not adequately 
described. On the other hand, animal models of collagenase-induced degenerative injuries revealed failed healing [2] and longstanding pain [3]. We believe that chronic tendinopathy is a result of failed healing of degenerative injuries (Figure 1). The reasons why the degenerative injuries fail to heal might be the core of the mystery. Since proinflammatory mediators affect various cellular activities related to tendon healing, it is possible that anti-inflammatory agents might negatively affect tendon healing and contribute to the development of tendinopathy.

\section{Non-steroidal anti-inflammatory drugs (NSAIDs)}

It is still a common practice to prescribe non-steroidal anti-inflammatory drugs (NSAIDs) which primarily work by inhibiting the production of pro-inflammatory prostaglandins. Interestingly, both beneficial and deleterious effects of NSAIDs on tendon healing were reported. It appears that NSAIDs exerted beneficial effects, if any, by influencing the remodeling of collagen matrix, resulting in reduction of cross-sectional area of the healing tendons but tensile strength may or may not be affected. NSAID may also negatively affect early tendon healing, as prostaglandin $\mathrm{E}_{2}\left(\mathrm{PGE}_{2}\right)$ is essential for early tendon healing such as control of vascular flow. We doubt that the unchecked use of NSAIDs may exert negative effects when prescribed to tendinopathy patients. As increased expression of cyclooxygenase 2 (COX-2) and increased produc-

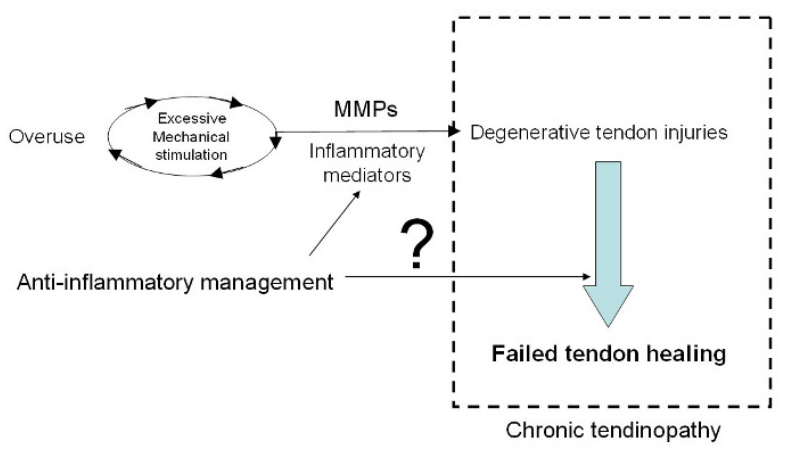

\footnotetext{
Figure I

Overuse or excessive mechanical stimulation to tendons can lead to elevation of metalloproteinases (MMPs) which mediate collagen degradation and hence degenerative tendon injuries. Healing responses are activated but failed to repair the degenerative injuries, resulting in tendinopathy. It is possible that anti-inflammatory management may affect the development of degenerative injuries as well as the pathological processes of failed healing in tendinopathy.
}

tion of $\mathrm{PGE}_{2}$ were found in chronic tendinopathy samples [4], the involvement of $\mathrm{PGE}_{2}$ for the development of chronic tendinopathy was implied. As $\mathrm{PGE}_{2}$ may mediate the development of overuse tendon injury by induction of metalloproteinases, it is possible that suppression of $\mathrm{PGE}_{2}$ may have reduced the extent of degenerative injuries but the normal matrix remodeling would also be affected, which may contribute to the failed tendon healing. Further investigation is urgently needed to disseminate the double-edged properties of prostaglandins in tendon healing before we could advocate the use of NSAIDs for chronic or acute tendon injuries.

\section{Corticosteroids}

Corticosteroids are strong anti-inflammatory agents and peri-tendinous injection of corticosteroid is commonly used to treat the chronic pain in tendinopathy. However, the use of corticosteroid may increase risk of spontaneous ruptures and the deleterious effects of corticosteroid were demonstrated on culture human tendon fibroblasts, including cell viability, proliferation and matrix synthesis [5]. In spite of its potential hazards, corticosteroid injections are still given indiscriminately in many sport clinics!! There is no doubt that the adverse effect of corticosteroids on tendon cells would affect the healing responses to degenerative injuries, corticosteroid injection should be considered as a last resort with careful control on the dosages.

\section{Other anti-inflammatory modalities}

Apart from NSAIDs and corticosteroids, the use of physical therapies to reduce inflammatory signs in tendon injuries is common, but rigorous scientific studies on the efficacy and the underlying mechanisms are not available. Pulsed electromagnetic fields and low level laser treatments may reduce inflammation, probably by suppressing $\mathrm{PGE}_{2}$ production. The therapeutic effect of extracorporeal shockwave therapy may be attributed to its anti-inflammatory actions through modulation of nitric oxide production. It appears that these biophysical interventions also exerted anti-inflammatory actions through modulation of pro-inflammatory mediators. As they are relatively safe, further exploration to consolidate their efficacies may yield a better clinical practice for anti-inflammatory management for tendon injuries.

It would be a breakthrough if we could identify disturbed prostaglandin levels as one of the causes of failed healing in tendinopathy, which will certainly guide the development of effective treatment strategies for overuse tendon injuries. In most clinical practice, it is very difficult to monitor the use of anti-inflammatory management for chronic tendinopathy, let alone restricting it in a rigid protocol. 


\section{References}

I. Millar NL, Wei AQ, Molloy T], Bonar F, Murrell GA: Cytokines and apoptosis in supraspinatus tendinopathy. J Bone Joint Surg $\mathrm{Br}$ 2009, 91 (3):417-24.

2. Lui PP, Fu SC, Chan LS, Hung LK, Chan KM: Chondrocyte phenotype and ectopic ossification in collagenase-induced tendon degeneration. J Histochem Cytochem 2009, 57(2):91-100.

3. Fu SC, Chan KM, Chan LS, Fong DT, Lui PY: The use of motion analysis to measure pain-related behaviour in a rat model of degenerative tendon injuries. I Neurosci Methods 2009, I79(2):309-18.

4. Fu SC, Wang W, Pau HM, Wong YP, Chan KM, Rolf CG: Increased expression of transforming growth factor- $\beta I$ in patellar tendinosis. Clin Orthop Rel Res 2002, 400: $174-183$.

5. Wong MWN, Tang YN, Fu SC, Lee KM, Chan KM: Triamcinolone Suppresses Human Tenocyte Cellular Activity and Collagen Synthesis. Clin Orthop Rel Res 2004, 42 I:277-28I.

Publish with Bio Med Central and every scientist can read your work free of charge

"BioMed Central will be the most significant development for disseminating the results of biomedical research in our lifetime. "

Sir Paul Nurse, Cancer Research UK

Your research papers will be:

- available free of charge to the entire biomedical community

- peer reviewed and published immediately upon acceptance

- cited in PubMed and archived on PubMed Central

- yours - you keep the copyright

Submit your manuscript here:

http://www.biomedcentral.com/info/publishing_adv.asp 